\title{
The clinical potential of blood-proteomics in multiple sclerosis
}

\author{
Roberto De Masi ${ }^{1,2,3^{*}}$, Sergio Pasca ${ }^{2,3}$, Rocco Scarpello $^{2}$, Adele Idolo ${ }^{4}$ and Antonella De Donno ${ }^{4}$
}

\begin{abstract}
Background: The aetiology of multiple sclerosis (MS) remains unknown. This hampers molecular diagnosis and the discovery of bio-molecular markers. Consequently, MS diagnostic procedures are complex and criteria for assessing therapeutic efficacy are controversial, suggesting that a pathophysiological rather than an aetiological approach to the disease would be more appropriate. In this regard, blood-proteomics represents a still-unexplored tool. We investigated the potential of proteomics as applied to peripheral blood mononuclear cells (PBMCs) for differentiating treatment-naive RR-MS patients from healthy controls and from IFN-treated RR-MS patients.
\end{abstract}

Methods: A comparative analysis of PBMC proteins isolated from 13 unselected IFN-treated RR-MS patients, 6 IFN-untreated RR-MS patients and 14 matched healthy controls was performed using two-dimensional gel electrophoresis and MALDI-TOF mass spectrometry. We considered the volume of each spot, expressed as a percentage of the total volume of all spots in the gel. Heuristic clustering was applied to a composite population made up of a random sequence of gels from the different groups in comparison. For the differentially expressed proteins, we applied the Student's $t$-test to identify only those down- or up-regulated at least 2.5-fold $[$ Ratio $(R) \geq 2.5]$ with respect to the homologous spots of the compared groups.

Results: Rho-GDI2, Rab2 and Cofilin1 were found to be associated with down-regulated and naïve group phenotypes; Cortactin and Fibrinogen beta-Chain Precursor were found to be associated with down-regulated and group-related IFN-treated RR-MS phenotypes. Thus, by means of similarity analysis, the proteomes were homogeneously segregated into three distinct groups corresponding to naive, IFN-treated and healthy control subjects. Interestingly, no separation was found between IFN-treated and healthy controls. Moreover, the molecular phenotypes were consistent with disease pathogenesis.

Conclusions: We demonstrated for the first time, albeit only with preliminary data, the aprioristic possibility of distinguishing naive and IFN-treated MS groups from controls, and naive from IFN-treated MS patients using a blood sample-based methodology (i.e. proteomics) alone. The functional profile of the identified molecules provides new pathophysiological insight into MS. Future development of these techniques could open up novel applications in terms of molecular diagnosis and therapy monitoring in MS patients.

Keywords: Multiple sclerosis, Blood-proteomics, Interferon therapy

\footnotetext{
* Correspondence: dmsrrt@gmail.com

'Laboratory of Neuroproteomics, Multiple Sclerosis Centre, Complex

Operative Unit of Neurology-Stroke Unit, "F. Ferrari" Hospital via

circonvallazione, 73042, Casarano, Lecce, Italy

${ }^{2}$ Complex Operative Unit of Neurology-Stroke Unit, "F. Ferrari" Hospital,

Casarano, Lecce, Italy

Full list of author information is available at the end of the article
} 


\section{Background}

Despite the extensive literature in the aetiological field, infectious and genetic theories have failed to identify the cause of the disease, and so multiple sclerosis (MS) is an autoimmune pathology whose aetiology is still unknown [1,2]. This is responsible for the lack of molecular diagnoses and therapy monitoring. On the other hand, there is increasingly consistent pathogenetical evidence that peripheral auto-reactive T-cells play a central role in provoking inflammatory demyelination and axonal loss in the brain parenchyma [3]. In addition, by acting on peripheral T-cells, interferon-beta (IFN- $\beta$ ) is thought to reduce disease activity, with an MRI-detectable effect in relapsing-remitting multiple sclerosis (RR-MS) patients [4-6], confirming the critical role of peripheral blood mononuclear cells (PBMCs) in CNS damage [7].

Molecular mimicry and epitope spreading further complicate aetiological investigation, suggesting that a pathophysiological rather than an aetiological approach to MS diagnosis may be more appropriate. Consequently, the MS-patient is diagnosed by pathophysiological evaluation of clinical or paraclinical dissemination in time and space [8-10], and the only biological tests currently considered to be of diagnostic relevance are oligoclonal band assessment and the exclusion of MS-mimicking conditions. Recently, proteomics has been successfully used to study autoimmune diseases by contextualizing the pathophysiological status of target cells (or tissues) with reference to their protein expression profile, the so-called molecular phenotype. This pathophysiologybased point of view may allow MS to be studied regardless of its aetiology. Dotzlaw H. and Schulz M. applied this technique to the PBMCs of rheumatoid arthritis patients and identified a differential fingerprint that separated diseased from healthy control subjects [11,12]. In addition, the resulting differentially expressed proteins helped to elucidate the molecular mechanisms of the disease. Blood-proteomics is also able to differentiate patients with Alzheimer's disease (AD) from healthy controls by providing a panel of plasma-proteins that also predicts progression to $\mathrm{AD}$ in preclinical patients affected by mild cognitive decline [13].

More recently, we found a functional correlation in MS patients between brain atrophy and the protein expression profile of PBMCs, confirming their pathophysiological involvement in disease evolution [14]. Despite the encouraging findings, to our knowledge, there has been no attempt to electively elucidate the diagnostic potential of PBMC-based proteomics in MS.

We assessed the potential of PBMC-based proteomic analysis applied to a random, blind population made up of IFN-untreated MS patients and healthy controls, differentiating and separating them into homogeneous groups. Secondarily we investigated the ability of the same methodology to blindly differentiate IFN-treated RR-MS patients from IFN-untreated (naïve) patients.

\section{Methods}

\section{Study population}

Three different classes of study population subjects were enrolled in a case-controlled cross-sectional proteomic study at the MS unit of "F. Ferrari" Hospital, Neurology Division, Casarano (Le), Italy:

1) Thirteen (seven male, six female) consecutive unselected IFN-treated RR-MS (RRt) patients taking 30 $\mu \mathrm{g}$ of interferon $\beta$-1a I.M. a week in accordance with the international guidelines of Jacob et al. [15].

2) Six (three male, three female) consecutive sex/age matched IFN-untreated RR-MS ( $R R u$, naïve) subjects.

All MS patients were previously diagnosed in accordance with standard criteria [10] and gave blood samples following a steroid- and exacerbation-free period of at least three months. Patients were selected for the study on the basis of the following criteria: (i) they were in the RR phase of the disease, classified in accordance with standard disease course criteria [16]; (ii) they were aged between 23 and 65; (iii) they had not undergone any immune-modulation therapy (naïve patients); (iv) they were prepared to express written informed consent. Patients who had been treated with immunosuppressive drugs or were affected by other comorbidity conditions were excluded from this study. Only non-interfering symptomatic therapy was tolerated.

3) Fourteen (seven male, seven female) age/sex matched healthy controls $(\mathrm{HC})$.

All subjects were enrolled and underwent blood sampling after expressing informed written consent. Approval of this study was provided by the local hospital and by the ethics committee of the Local Health Authority of Lecce (ASL/LE).

\section{PBMC isolation and lysis}

Heparinized blood $(15 \mathrm{ml})$ was collected by venipuncture and immediately processed. First, blood was centrifuged at $150 \times \mathrm{g}$ for $5 \mathrm{~min}$ and the platelet-enriched supernatant was removed. The pellet was then diluted with PBS (Phosphate Buffered Saline) solution and layered on a Ficoll density gradient (GE Healthcare). Subsequently, PBMCs were isolated by centrifugation $(400 \times \mathrm{g}$ for $40 \mathrm{~min})$ and processed within $1 \mathrm{~h}$. After isolation, the lympho-monocytes were washed twice in PBS and lysed in $0.5 \mathrm{ml}$ of sample buffer (CHAPS) and protease inhibitor cocktail. To minimize nucleic acid interference with protein 
migration, the sample was sonicated 3 times $\mathrm{x} 5 \mathrm{sec}$ on ice (low amplitude). Finally, protein concentration was determined by Bradford's method (Bio-Rad Protein assay).

\section{Two-dimensional gel electrophoresis First dimension run}

Sample loading: $80 \mu \mathrm{g}$ of proteins were mixed with rehydration solution (7 M Urea, $2 \mathrm{M}$ thiourea, 4\% CHAPS, $50 \mathrm{mM}$ DTT and 0.8\% IPG Buffer) to obtain a final volume of $250 \mu \mathrm{l}$. The samples were incubated for $30 \mathrm{~min}$ at $4{ }^{\circ} \mathrm{C}$ and applied to $13 \mathrm{~cm}$ IPG pH 4-7 gel strips (Amersham Biosciences).

Isoelectric focusing (IEF): IEF was performed with an IPGphor system (GE Healthcare) in 7 steps by gradually increasing the voltage after rehydration for $4 \mathrm{~h}: 30 \mathrm{~V}$ for $12 \mathrm{~h}$, from $30 \mathrm{~V}$ to $500 \mathrm{~V}$ in $30 \mathrm{~min}, 500 \mathrm{~V}$ for $1 \mathrm{~h}$, from $500 \mathrm{~V}$ to $1000 \mathrm{~V}$ in $30 \mathrm{~min}, 1000 \mathrm{~V}$ for $1 \mathrm{~h}$, gradient from $1000 \mathrm{~V}$ to $8000 \mathrm{~V}$ in $30 \mathrm{~min}, 8000 \mathrm{~V}$ for $2 \mathrm{~h}$, making a total of 20,617 Vh.

Equilibration: after IEF, the strips were equilibrated in 6 M Urea, 50 Mm Tris- $\mathrm{HCl}$ (pH 8.8), 30\% glycerol, 2\% SDS and $10 \mathrm{mg} / \mathrm{ml} \mathrm{DTT} \mathrm{for} 18 \mathrm{~min}$, followed by $25 \mathrm{mg} / \mathrm{ml}$ iodoacetamide for $10 \mathrm{~min}$.

\section{Second dimension run}

The second dimension was performed on a 9-16\% gradient SDS-polyacrylamide gel $(16 \times 18 \mathrm{~cm}, 1.5 \mathrm{~mm}$ thick $)$ and run at a constant current of $28 \mathrm{~mA} /$ gel at $8^{\circ} \mathrm{C}$ until the $\mathrm{BPB}$ reached the anodic end of the gel. Wide range protein standard (Sigma-Aldrich) was loaded together with the sample.

Development and staining: proteins were visualized by ammoniacal silver staining, adding thiosulfate after the gel polymerization step [17]. Each sample was run in duplicate, making a total of sixty-six gels subjected to 2-D electrophoresis.

\section{Image and statistical analysis}

Gel images were acquired using an Image Scanner (GE Healthcare), with a scanning resolution of 300 dpi. Spot detection, matching and data analysis were performed with Image MasterTM 2D Platinum software, version 5.0 (GE Healthcare). The gels were divided into three classes (healthy controls, IFN-untreated RR-MS patients and IFN-treated RR-MS patients) and a reference gel with the highest number of spots was created for each class. The software was then used to detect and match spots between gels by the matching method included in the software package. Spot detection and spot matches were corrected manually where necessary. The analysis was carried out by comparing the volume of each spot expressed as a percentage of the total volume of all spots in the gel.

Heuristic clustering was used on a random population composed of a blind sequence of gels from the classes in comparison. This analysis assigned each gel to a homogenous subclass depending on the similarity between each spot and its homologue in all the other gels in comparison. The Student's $t$-test was applied to the differentially expressed proteins in order to identify only those down- or up-regulated at least 2.5-fold $[\operatorname{Ratio}(R) \geq 2.5]$ with respect to homologous spots of the compared groups. In addition, Leven's test was applied to every differentially expressed spot to assess the similarity of variance between the compared groups.

Cluster analysis was applied first to a composite population made up of naive patients and healthy controls, and then to a composite population made up of IFNtreated and untreated RR-MS patients. In both cases we used a random blind sequence of gels.

\section{Protein identification}

For preparative mass spectrometry analysis, gels were stained with MS-compatible silver staining or with Blue Silver colloidal Coomassie $[18,19]$.

Silver stained spots were excised from preparative gels and destained with hydrogen peroxide and ammonium bicarbonate as reported by Sumner et al. [20]. Coomassie stained spots were processed by incubating with $50 \mu \mathrm{l}$ of $50 \mathrm{mM} \mathrm{NH}_{4} \mathrm{HCO}_{3}$ in $40 \%$ ethanol until full decolouration. The spots were then incubated with $50 \mu \mathrm{l}$ of acetonitrile for $10 \mathrm{~min}$. The dried gel pieces were rehydrated in $16 \mu \mathrm{l}$ $(20 \mu \mathrm{g} / \mathrm{ml})$ of trypsin solution (Sigma) and digested overnight at $37^{\circ} \mathrm{C}$.

Table 1 Clinical and demographic features of treated MS (RRt), untreated MS (RRu) and healthy control (HC) subjects

\begin{tabular}{|c|c|c|c|c|c|c|c|c|c|c|}
\hline & \multicolumn{3}{|c|}{ RRt $(n=16)$} & \multicolumn{3}{|c|}{$H C(n=12)$} & \multicolumn{3}{|c|}{$\mathrm{RRu}(\mathrm{n}=6)$} & \multirow[t]{2}{*}{$p$ value } \\
\hline & range & mean & SD & range & mean & SD & range & mean & SD & \\
\hline DISEASE DURATION & $2.0-14.0$ & 7.43 & 4.5 & & & & $2.9-13.9$ & 7.83 & 3.9 & $p=0.15$ \\
\hline AGE AT STUDY & $23.0-65.0$ & 42.6 & 11.1 & $22.2-64.3$ & 41.8 & 10.6 & $20.9-66.0$ & 42.9 & 12.2 & $p^{1,2,3} \geq 0.22$ \\
\hline EDSS & $0.0-5.0$ & 2.0 & 1.7 & & & & & & & \\
\hline SEX RATIO (O/Ő) & & $\% / 44 \%$ & & & $\% / 42 \%$ & & & $\% / 43$ & & \\
\hline
\end{tabular}

$p^{1,2,3}: p$ value of comparison between $\mathrm{HC} / \mathrm{RRt}$, RRu/RRt, RRu/HC groups respectively. 


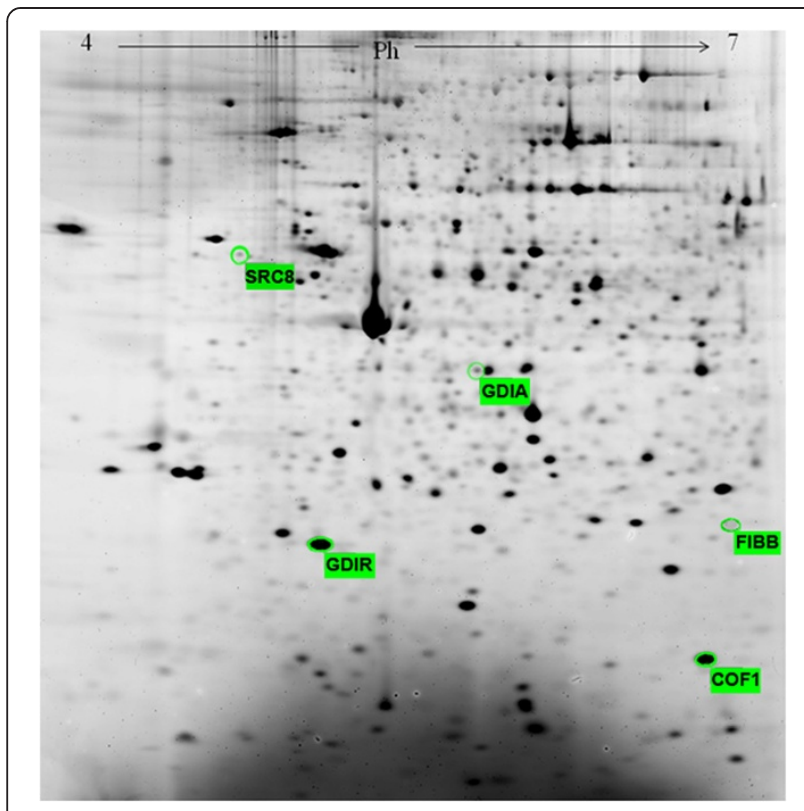

Figure 1 Reference map of PBMCs. Spots showing differential expression are indicated.

The peptide extract was desalted and concentrated by reverse phase extraction using ZipTip C18 microcolumn (Millipore, CA, USA). Peptides were directly eluted with $2 \mu \mathrm{l}$ of $\alpha$-cyano-4-hydroxycinnamic acid in 33\% acetonitrile, TFA $0.1 \%$, on a ground steel MTP 384 target (Bruker Daltonics).

MALDI-TOF analyses were performed on a Reflex IV mass spectrometer (Bruker Daltonics) in reflectron positive ion mode after internal and external calibration of the spectrum. The MALDI mass spectrum was externally calibrated with the peptide standard calibration II mixture (Bruker Daltonics) and then internally calibrated with trypsin autodigestion products $(842.51 \mathrm{~m} / \mathrm{z}, 1045.56 \mathrm{~m} / \mathrm{z}$ and $2239.14 \mathrm{~m} / \mathrm{z}$ ). Analyses were carried out in the mass range from 700 to $4000 \mathrm{~m} / \mathrm{z}$ and about 300 laser shots were taken for each spectrum.

Database searches were conducted with the MS-Fit or Mascot programs against Swiss-Prot and NCBI databases with the following parameters: human species, mass tolerance of $50 \mathrm{ppm}$, one missed cleavage site for tryptic peptides, allowing the modification of carbamidomethylation (C) and variable oxidation (M).

\section{Results}

Demographic characteristics were comparable in the diseased and control groups (Table 1). The mean duration of IFN-based therapy at the start of the study was 7 years. Figure 1 shows a reference map of PBMCs where all the spots showing differential expression are indicated. A list of them with their Mascot identification data is shown in Table 2 .

The heuristic clustering dendrogram indicated complete separation between the gels of healthy controls and those of diseased IFN-untreated patients (Figure 2); complete separation was also obtained between the gels of IFNuntreated and IFN-treated patients (Figure 3). These results were obtained by applying cluster analysis to one population composed of a random blind sequence of all the gels of $\mathrm{HC}$ and RRu subjects and another composed of all the gels from the $R R u$ and RRt patients.

In the first case, the differentially expressed proteins were: Rho-GDI2, Rab GDI $\beta$ (Rab2) and Cofilin 1. In the second case, the differentially expressed proteins were: Cortactin and the Fibrinogen $\beta$ Chain Precursor. Specifically, Rho GDI2, Rab2 and Cofilin were found to be downexpressed in IFN-untreated patients in comparison to healthy controls, while Cortactin and Fibrinogen $\beta$ chain precursor were found to be up-regulated in the untreated group compared to the treated group. Moreover, equality of variance was found in all the compared groups, except for Cofilin between the IFN-treated and untreated groups ( $\mathrm{R}$ and Leven's tests in Table 3 ). Figure 4 shows the shape of the most representative spots.

Lastly, clustering analysis applied to a population made up of a random blind sequence of all the gels from the RRt patients and $\mathrm{HC}$ subjects showed no homogeneous class separation.

All the differentially expressed spots sequenced by MALDI-TOF analysis exhibited $\mathrm{R} \geq 2.5(p \leq 0.001)$.

\section{Discussion}

This study assessed the aprioristic potential of proteomics for discriminating between RR-MS subjects and healthy controls and between IFN-treated RR-MS patients and untreated patients. Blind heuristic clustering applied to a random population of PBMC gels from IFN-treated and untreated MS patients was able to separate treated from naïve subjects and the latter from healthy controls.

Table 2 Identified proteins and corresponding Mascot score

\begin{tabular}{|c|c|c|c|c|c|c|c|c|c|}
\hline Entry name & Peptide matches & Swiss-Prot AC number & Description & pl & Mw & pl & Mw & Coverage & Score Mascot \\
\hline COF1 & 8 & P23528 & Cofilin 1 (non-muscle isoform) & 8.3 & 18371 & 5.91 & 19 & $57 \%$ & 107 \\
\hline SRC8 & 22 & Q14247 & Src substrate cortactin (Amplaxin) & 5.2 & 61637 & 5.08 & 85 & $35 \%$ & 207 \\
\hline FIBB & 31 & P02675 & Fibrinogen beta chain precursor & 8.5 & 55929 & 6.03 & 42 & $54 \%$ & 227 \\
\hline GDIR & 10 & P52565 & Rho GDP-dissociation inhibitor 1 & 5.0 & 23076 & 4.96 & 26 & $39 \%$ & 88 \\
\hline GDIA & 25 & P31150 & Rab GDP dissociation inhibitor alpha & 5.0 & 50583 & 4.99 & 59 & $52 \%$ & 304 \\
\hline
\end{tabular}




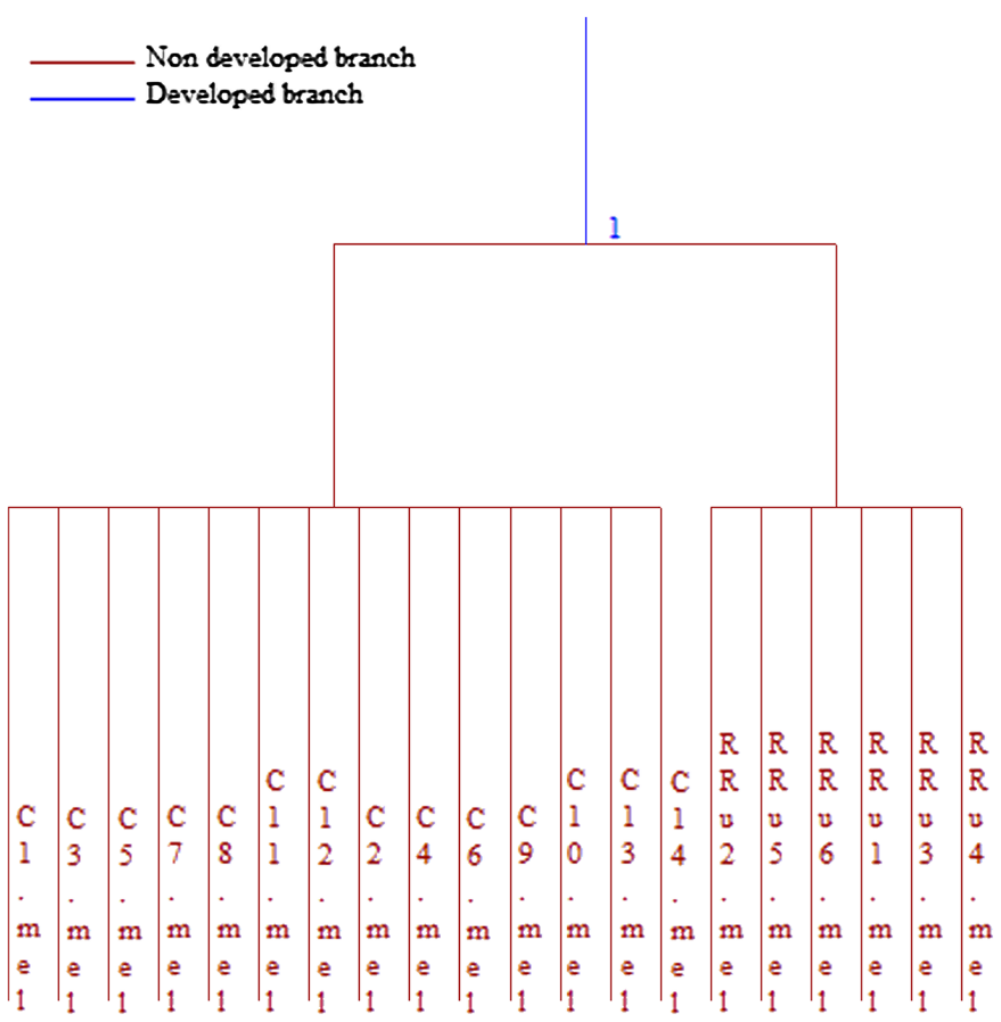

Figure 2 Dendrogram indicating complete separation between gels from healthy controls and IFN-untreated patients.

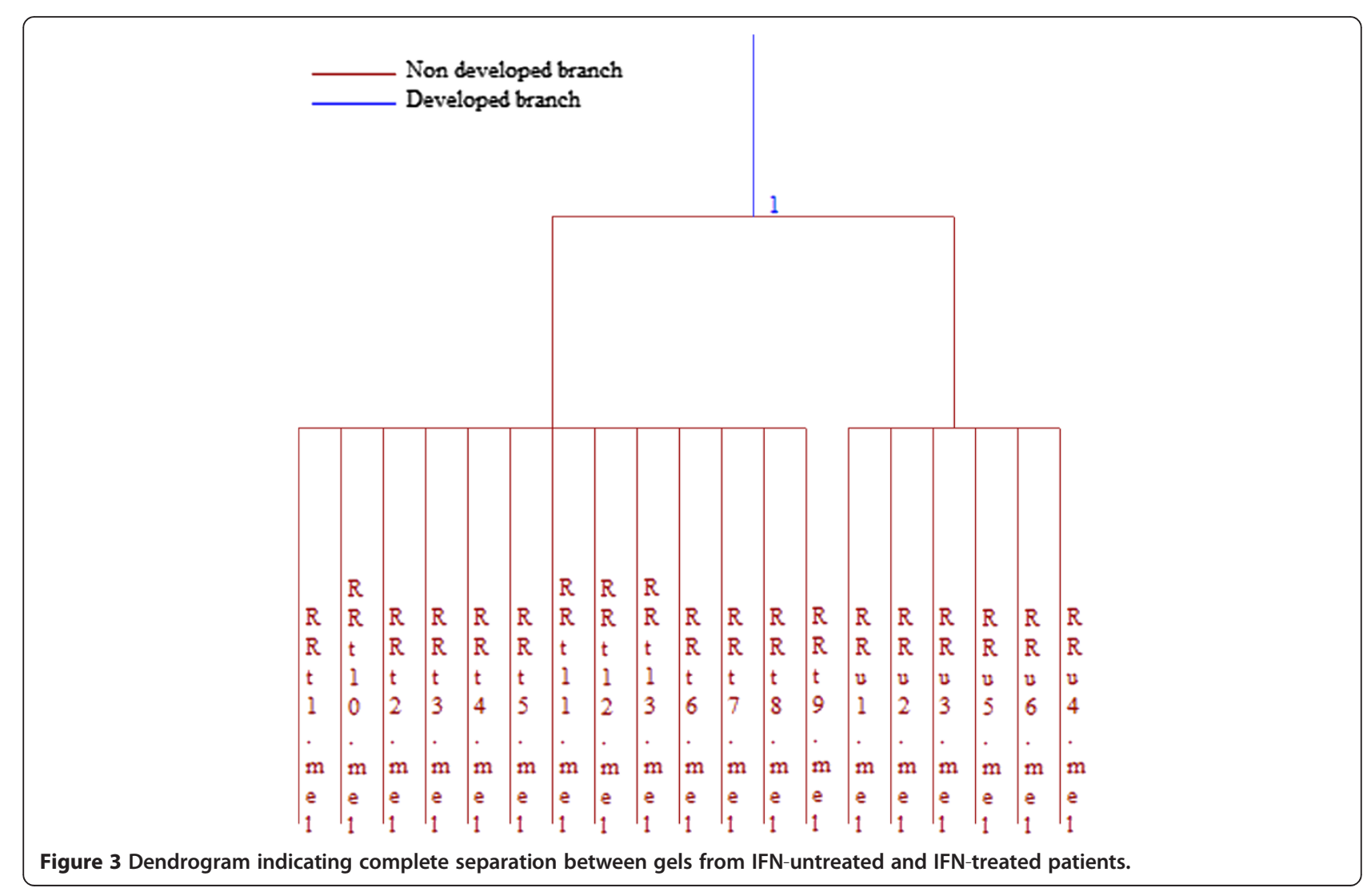


Table 3 Differentially expressed proteins with \% volume and related statistical parameters of treated MS (RRt), untreated MS (RRu) and healthy control (HC) subjects

\begin{tabular}{|c|c|c|c|c|c|c|c|}
\hline Entry name & $\begin{array}{c}\text { Average } \\
\text { Vol\% RRu } \\
\text { (SD; range) }\end{array}$ & $\begin{array}{c}\text { Average } \\
\text { Vol\% HC } \\
\text { (SD; range) }\end{array}$ & $\begin{array}{c}\text { Average } \\
\text { Vol\% RRt } \\
\text { (SD; range) }\end{array}$ & $\begin{array}{c}\text { RRu change } \\
\text { with respect to } \\
\text { HC (Vol\% ratio; } p \text { ) }\end{array}$ & $\begin{array}{l}\text { RRu change with } \\
\text { respect to RRt } \\
\text { (Vol\% ratio; } p \text { ) }\end{array}$ & $\begin{array}{c}\text { Levene's test } \\
\text { for equality of } \\
\text { variance (RRu/HC) }\end{array}$ & $\begin{array}{c}\text { Levene's test } \\
\text { for equality of } \\
\text { variance (RRt/RRu) }\end{array}$ \\
\hline Rho GDI2 & $\begin{array}{c}0.7340 \\
(0.26 ; 0.60)\end{array}$ & $\begin{array}{c}1.9738 \\
(0.28 ; 0.95)\end{array}$ & $/ /$ & Down 2.8; $(p=0.000)$ & $/ /$ & n.s. & $/ /$ \\
\hline Rab GDIß (Rab2) & $\begin{array}{c}0.2883 \\
(0.04 ; 0.12)\end{array}$ & $\begin{array}{c}0.0976 \\
(0.05 ; 0.19)\end{array}$ & $/ /$ & Down 2.9; $(p=0.000)$ & $/ /$ & n.s. & $/ /$ \\
\hline Cofilin 1 & $\begin{array}{c}0.1946 \\
(0.10 ; 0.24)\end{array}$ & $\begin{array}{c}0.9651 \\
(0.48 ; 1.83)\end{array}$ & $/ /$ & Down 4.9; $(p=0.000)$ & $/ /$ & $p=0.043$ & $/ /$ \\
\hline Cortactin & $\begin{array}{c}0.3912 \\
(0.04 ; 0.11)\end{array}$ & $/ /$ & $\begin{array}{c}0.0546 \\
(0.04 ; 0.13)\end{array}$ & $/ /$ & $\begin{array}{c}\text { Up 7.1; } \\
(p=0.000)\end{array}$ & $/ /$ & n.s. \\
\hline $\begin{array}{c}\text { Fibrinogen } \beta \\
\text { Chain Precursor }\end{array}$ & $\begin{array}{c}0.1372 \\
(0.02 ; 0.08)\end{array}$ & $/ /$ & $\begin{array}{c}0.0039 \\
(0.01 ; 0.03)\end{array}$ & $/ /$ & $\begin{array}{l}\text { Up 35.1; } \\
(p=0.000)\end{array}$ & $/ /$ & n.s. \\
\hline
\end{tabular}

n.s.: not significant.

Specifically, Rho-GDI2, Rab GDI $\beta$ (Rab2) and Cofilin 1 were found to be down-regulated in naïve patients, and Cortactin and Fibrinogen $\beta$ Chain Precursor were found to be down-regulated in the IFN-treated group. Each group of gels belonging to a dendrogram branch was separated from the other branches by the presence of differentially expressed spots exhibiting two key features: the non-overlapping mean \% volume values and the large difference between the mean values (at least two and a half times the mean absolute value) of the \% volume of each sub-group.

Although these findings represent preliminary data, they demonstrate for the first time the concrete role of proteomics in the molecular diagnosis of MS. Moreover, proteomics is able to distinguish the PBMC profile of an IFN-treated patient from that of a naive one. This latter finding may seem to be of low importance, but it could contribute to the molecular characterization of patients who do not respond to interferon therapy.

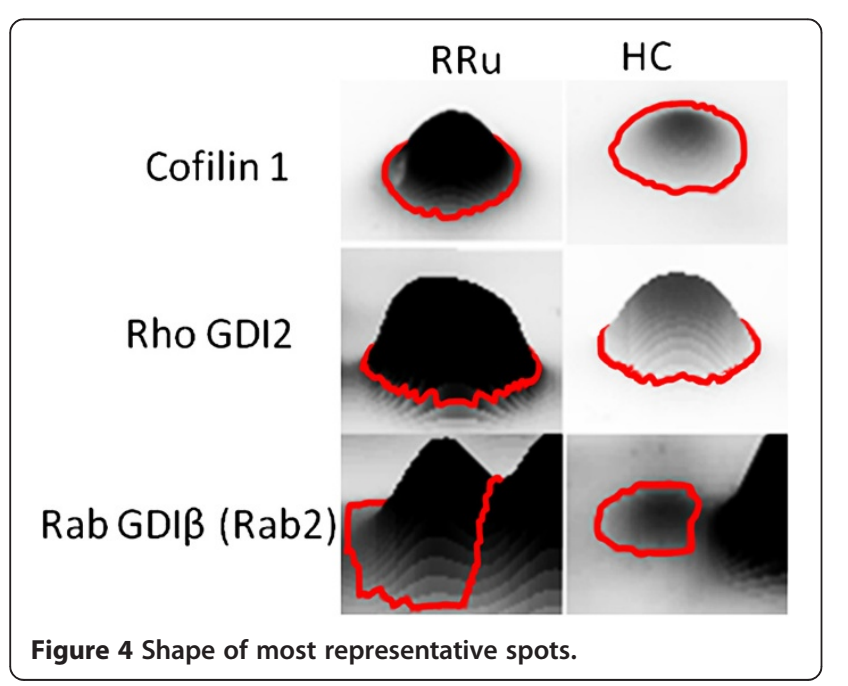

In addition, our data confirmed, at a molecular level, the disregulation and consequent diversion of the immune system to the "self" in MS patients. We also found quantitative rather than qualitative differences between the PBMC profile of naïve patients and that of healthy controls. This is in line with our previous work, which did not find a differential pattern in MS. The application of cluster analysis in the present study is seen to have a diagnostic value based on a pathophysiological methodology, and the differentially expressed proteins identified here exhibit a coherent functional profile in the pathophysiological context of MS.

Rho-GDI2 and Rab GDI $\beta$ (Rab2) are key GTPase inhibitors: they regulate a group of high-level proteins in cell biology and are involved in various fundamental cellular events including secretion, proliferation, intracellular signalling, cytokinesis, vesicular trafficking and lymphocyte extravasation through blood-CNS barriers.

Three general classes of GTPase signalling regulators have been identified: guanine nucleotide exchange factors (GEFs), GTPase-activating proteins (GAPs) and guanine nucleotide dissociation inhibitors (GDIs).

Rho-GDI proteins form a large complex, with the Rho protein helping to prevent diffusion within the membrane and the cytosol [21]. Rho GTPases at the plasma membrane are in turn known to be associated with TCR cascades following antigen recognition in the presence of MHC molecules. This association is developed by the ZAP-70 protein. A substrate for its activity is the adapter molecule LAT (linker for activation of T cells), linked to the Rho/Rac domain in a multi-protein signalling complex that includes PLC $\gamma$, Grb2 and Ras. They are the main effectors for intracellular actin dynamics and gene expression. Interestingly, there is much evidence to indicate that Rho GTPase is a critical signalling intermediate in vascular inflammation and inflammatory injury.

Rab2 proteins are monomeric GTPases specializing in vesicular trafficking, as their sub-cellular localization in 
the cis-Golgi lattice suggests. Moreover, they can interact with the SNARE system, thereby coupling membrane cohesion to their fusion.

It thus seems clear that GDI represents a pivotal regulator of the major cellular functions that support the same abnormal mechanisms postulated in MS pathogenesis. The GDI down-expression in the PBMCs of naive MS patients is evidence for the basal inflammatory activity of leukocytes, as expected in a chronic inflammatory disease. Consistently, GDI down-expression was not seen in the IFN-treated MS group. Indeed, the anti-inflammatory effects of $\beta$-interferon, with notable reduction in cell trafficking, secretion, proliferation and leukocyte extravasation, are well known. Likewise, Cofilin, a ubiquitous eukaryotic actin-binding protein with actin-severing activity, was seen to be down-expressed in the IFN-treated group.

The down-expression of Cortactin and the Fibrinogen $\beta$ Chain Precursor in the treated MS-group again suggests a pharmacological effect. Indeed, the former, which is over-expressed in human carcinoma cells, is implicated in the modulation of cellular adhesion; the latter is an extracellular protein that stimulates leukocyte functional activity and adhesion during inflammation [22-24].

Thus we argue that naïve RR-MS is characterized by chronic, basal activation of the fundamental proinflammatory mechanisms correlated with Rho and Rab2 GDI de-inhibition. This condition does not seem to characterise IFN-treated patients, as also suggested by the decrease in Cortactin and Fibrinogen beta chain precursor in the RRt group.

In the literature, the main proteomic results have been obtained in neoplasmic, genetic and infectious diseases that are characterized by aberrant molecular phenotypes such as disease-specific differentially expressed proteins. In our study, rather than aberrant phenotypes we found aberrant expression of normal proteins. Our differentially expressed proteins thus do not appear to indicate pathological specificity, but reflect the functional condition of PBMCs.

One weakness of our study is the limited population, but two other elements need to be considered: with the sole exception of Cofilin, the low and homogenous magnitude of inter-group and intra-group data dispersion confirms the accuracy of the method; the difference in protein expression over the third standard deviation makes the study fairly robust.

\section{Conclusions}

Although our statements refer to a population already screened for other MS-like conditions, we can conclude that PBMC proteomics coupled with heuristic clustering is able to distinguish treatment-naïve MS patients from healthy control subjects and IFN-treated patients from untreated patients.
Lastly, Rho- and Rab2-GDI inhibitors, Cofilin, Cortactin and the Fibrinogen beta chain precursor can improve our knowledge of the pharmacodynamics of $\beta$-interferon and the pathophysiology of MS. Although further confirmation by means of routine-based techniques is warranted, they should be considered as potentially clinically relevant signalling factors in the laboratory criteria of IFN-responding RR-MS patients and in the early diagnosis of the disease.

\section{Abbreviations}

MS: Multiple sclerosis; PBMCs: Peripheral blood mononuclear cells; IFN- $\beta$ : Interferon-beta; RR-MS: Relapsing-remitting multiple sclerosis; AD: Alzheimer's disease; RRt: RR IFN-treated; RRu: RR IFN-untreated; HC: Healthy controls; PBS: Phosphate Buffered Saline; IEF: Isoelectric focusing; GEFs: Guanine nucleotide exchange factors; GAPs: GTPase-activating proteins; GDls: Guanine nucleotide dissociation inhibitors.

\section{Competing interests}

The Authors declare that they have no competing interests.

\section{Authors' contributions}

RDM was involved in study design, data acquisition, data analysis, manuscript drafting, literature research, statistical analysis and clinical studies. SP was involved in study design, data acquisition, data analysis, manuscript drafting, literature research and clinical studies. RS was involved in study design, data acquisition, data analysis, manuscript drafting, literature research and clinical studies. Al performed data acquisition and literature research. ADD drafted the manuscript and performed literature research. All authors read and approved the final manuscript.

\section{Acknowledgements}

The authors wish to thank Dr Anna Maria Paolini, Managing Director of the "F. Ferrari" Hospital in Casarano, for her unconditional general and economic support. Moreover we thank Dr Stefania Citiso for providing technical support.

\section{Author details}

${ }^{1}$ Laboratory of Neuroproteomics, Multiple Sclerosis Centre, Complex Operative Unit of Neurology-Stroke Unit, "F. Ferrari" Hospital via circonvallazione, 73042, Casarano, Lecce, Italy. ${ }^{2}$ Complex Operative Unit of Neurology-Stroke Unit, "F. Ferrari" Hospital, Casarano, Lecce, Italy. ${ }^{3}$ Multiple Sclerosis Center, "F. Ferrari" Hospital, Casarano, Lecce, Italy. ${ }^{4}$ Department of Biological and Environmental Sciences and Technologies, Lab of Hygiene, University of Salento, Lecce, Italy.

Received: 5 March 2013 Accepted: 3 May 2013

Published: 21 May 2013

\section{References}

1. Giovannoni G, Ebers G: Multiple sclerosis: the environment and causation. Curr Opin Neurol 2007, 20:261-268.

2. Comabella M, Khoury SJ: Immunopathogenesis of multiple sclerosis. Clin Immunol 2012, 142:2-8.

3. Sospedra M, Martin R: Immunology of multiple sclerosis. Annu Rev Immunol 2005, 23:683-747.

4. Richert ND, Howard T, Frank JA, Stone R, Ostuni J, Ohayon J, Bash C, McFarland HF: Relationship between inflammatory lesions and cerebral atrophy in multiple sclerosis. Neurology 2006, 66:551-556.

5. Rudick RA, Goelz SE: Beta-interferon for multiple sclerosis. Exp Cell Res 2011, 317(9):1301-1311.

6. Dhib-Jalbut $S$, Steven Marks MD: Interferon- $\beta$ mechanisms of action in multiple sclerosis. Neurology 2010, 74(Suppl 1):S17-S24.

7. Patanella AK, Zinno M, Quaranta D, Nociti V, Frisullo G, Gainotti G, Tonali PA, Batocchi AP, Marra C: Correlations between peripheral blood mononuclear cell production of BDNF, TNF-alpha, IL-6, IL-10 and cognitive performances in multiple sclerosis patients. J Neurosci Res 2010, 88:1106-1112.

8. McDonald WI, Compston A, Edan G, Goodkin D, Hartung HP, Lublin FD, McFarland HF, Paty DW, Polman CH, Reingold SC, Sandberg-Wollheim M, Sibley W, Thompson A, van den Noort S, Weinshenker BY, Wolinsky JS: 
Recommended diagnostic criteria for multiple sclerosis: guidelines from the International Panel on the Diagnosis of Multiple Sclerosis. Ann Neurol 2001, 50:121-127

9. Polman $\mathrm{CH}$, Reingold SC, Edan G, Filippi M, Hartung HP, Kappos L, Lublin FD, Metz LM, McFarland HF, O'Connor PW, Sandberg-Wollheim M, Thompson AJ, Weinshenker BG, Wolinsky JS: Diagnostic criteria for multiple sclerosis: 2005 revisions to the "McDonald Criteria". Ann Neurol 2005, 58:840-846.

10. Polman CH, Reingold SC, Banwell B, Clanet M, Cohen JA, Filippi M, Fujihara K, Havrdova E, Hutchinson M, Kappos L, Lublin FD, Montalban X, O'Connor PW, Sandberg-Wollheim M, Thompson AJ, Waubant E, Weinshenker BG, Wolinsky JS: Diagnostic criteria for multiple sclerosis, Revisions to the McDonald criteria. Ann Neurol 2010, 2011 (69):292-302.

11. Dotzlaw H, Schulz M, Eggert M, Neeck G: A pattern of protein expression in peripheral blood mononuclear cells distinguishes rheumatoid arthritis patients from healthy individuals. Biochim Biophys Acta 2004, 1696:121-129.

12. Schulz M, Dotzlaw H, Mikkat S, Eggert M, Neck G: Proteomic Analysis of Peripheral Blood Mononuclear Cells: Selective Protein Processing Observed in Patients with Rheumatoid Arthiritis. J Proteome Res 2007. Publication date on Web 17/08/2007.

13. Britschgi M, Wyss-Coray $\mathrm{T}$ : Blood protein signature for the early diagnosis of Alzheimer disease. Arch Neurol 2009, 66(2):161-165.

14. De Masi R, Vergara D, Pasca S, Acierno R, Greco M, Spagnolo L, Blasi E, Sanapo F, Trianni G, Maffia M: PBMCs protein expression profile in relapsing IFN-treated multiple sclerosis: a pilot study on relation to clinical findings and brain atrophy. J Neuroimmunol 2009, 210:80-86.

15. Jacobs LD, Cookfair DL, Rudick RA, Herndon RM, Richert JR, Salazar AM, Fischer JS, Goodkin DE, Granger CV, Simon JH, Alam JJ, Bartoszak DM, Bourdette DN, Braiman J, Brownscheidle CM, Coats ME, Cohan SL, Dougherty DS, Kinkel RP, Mass MK, Munschauer FE 3rd, Priore RL, Pullicino PM, Scherokman BJ, Weinstock-Guttman B, Whitham RH: Intramuscular interferon beta-1a for disease progression in relapsing multiple sclerosis. Ann Neurol 1996, 39(3):258-294.

16. Lublin FD, Reingold SC: Defining the clinical course of multiple sclerosis: results of an international survey. Neurology 1996, 46:907-911.

17. Hochstrasser DF, Merril CR: 'Catalysts' for polyacrylamide gel polymerization and detection of proteins by silver staining. Appl Theor Electrophor 1988, 1:35-40.

18. Mortz E, Krogh TN, Vorum H, Görg A: Improved silver staining protocols for high sensitivity protein identification using matrix-assisted laser desorption/ionization-time of flight analysis. Proteomics 2001, 1:1359-1363.

19. Candiano G, Bruschi M, Musante L, Cantucci L, Ghiggeri GM, Carnemolla B, Orecchia P, Zardi L, Rigetti PG: Blue silver: a very sensitive colloidal Coomassie G-250 staining for proteome analysis. Electrophoresis 2004, 25:1327-1333.

20. Sumner LW, Wolf-Summer B, White SP, Asirvatham VS: Silver stain removal using $\mathrm{H}_{2} \mathrm{O} 2$ for enhanced peptide mass mapping by matrix-assisted laser desorption/ionization time-of-flight mass spectrometry. Rapid Commun Mass Spectrom 2002, 16:160-168.

21. Ellenbroek S, Collard J: Rho GTPases: functions and association with cancer. Clin Exp Metastasis 2007, 24(8):657-672.

22. Zhambalova BA, Azizova OA, Lopukhin YM: Effect of fibrinogen on functional activity of blood leukocytes. Bull Exp Biol Med 2002, 133(5):448-449.

23. Languino LR, Plescia J, Duperray A, Brian AA, Plow EF, Geltosky JE, Altieri DC: Fibrinogen mediates leukocyte adhesion to vascular endothelium through an ICAM-1-dependent pathway. Cell 1993, 73:1423-1434.

24. Pearson MJ, Lipowsky HH: Effect of Fibrinogen on Leukocyte Margination and Adhesion in Postcapillary Venules. Microcirculation 2004, 11(3):295-306.

doi:10.1186/1471-2377-13-45

Cite this article as: De Masi et al:: The clinical potential of bloodproteomics in multiple sclerosis. BMC Neurology 2013 13:45.

\section{Submit your next manuscript to BioMed Central and take full advantage of:}

- Convenient online submission

- Thorough peer review

- No space constraints or color figure charges

- Immediate publication on acceptance

- Inclusion in PubMed, CAS, Scopus and Google Scholar

- Research which is freely available for redistribution

Submit your manuscript at www.biomedcentral.com/submit 